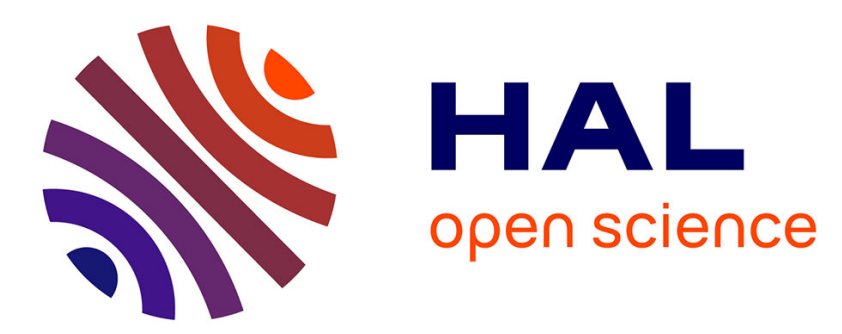

\title{
Cognitive and affective Theory of Mind in mild to moderate Alzheimer's disease.
}

Mickael Laisney, Laetitia Bon, Catherine Guiziou, Nathalie Daluzeau, Francis Eustache, Béatrice Desgranges

\section{- To cite this version:}

Mickael Laisney, Laetitia Bon, Catherine Guiziou, Nathalie Daluzeau, Francis Eustache, et al.. Cognitive and affective Theory of Mind in mild to moderate Alzheimer's disease.. Journal of neuropsycholgy, 2013, 7 (1), pp.107-20. 10.1111/j.1748-6653.2012.02038.x . inserm-00707840

HAL Id: inserm-00707840 https://www.hal.inserm.fr/inserm-00707840

Submitted on 25 Oct 2012

HAL is a multi-disciplinary open access archive for the deposit and dissemination of scientific research documents, whether they are published or not. The documents may come from teaching and research institutions in France or abroad, or from public or private research centers.
L'archive ouverte pluridisciplinaire HAL, est destinée au dépôt et à la diffusion de documents scientifiques de niveau recherche, publiés ou non, émanant des établissements d'enseignement et de recherche français ou étrangers, des laboratoires publics ou privés. 


\title{
Cognitive and affective theory of mind in mild to moderate Alzheimer's disease
}

Mickaël LAISNEY, Ph.D. ${ }^{1,2,3,4}$, Laetitia BON, M.Sc. ${ }^{1,2,3,4}$, Catherine GUIZIOU, M.Sc. ${ }^{5}$, Nathalie DALUZEAU, M.D. ${ }^{5}$, Francis EUSTACHE, Ph.D. ${ }^{1,2,3,4}$ and Béatrice DESGRANGES, Ph.D. ${ }^{1,2,3,4}$

1 INSERM, U1077, Caen, France

2 Université de Caen Basse-Normandie, UMR-S1077, Caen, France

3 Ecole Pratique des Hautes Etudes, UMR-S1077, Caen, France

4 CHU de Caen, U1077, Caen, France

5 Centre Hospitalier Robert Bisson, Lisieux, France

Corresponding author: Béatrice Desgranges

Laboratoire de Neuropsychologie Inserm-EPHE-UCBN U1077, CHU de CAEN, F-14033

CAEN; Tel.: +33 (0)231 065 195; E-mail address: desgranges-b@ chu-caen.fr

\begin{abstract}
Theory of mind (ToM) allows one's own and others' cognitive and emotional mental states to be inferred. Although many patients with Alzheimer's disease (AD) display impaired social functioning as their disease progresses, very few studies have investigated ToM in AD. Those that have done so suggest that patients' ToM deficits are the consequence of other cognitive impairments. The aim of this study was thus to investigate changes in both the cognitive and the affective dimensions of ToM in $\mathrm{AD}$, using tasks designed to circumvent the patients' comprehension difficulties. Sixteen mild to moderate AD patients and 15 healthy controls matched on age, sex and education level underwent cognitive (preference judgment and first- and second-order false belief) and affective (Reading the Mind in the Eyes) ToM assessments. Comprehension of false belief stories was verified and an additional neuropsychological examination was undergone. We observed impaired performances by AD patients on all the ToM tasks. While working memory and executive functioning impairments contributed to the deterioration in the more complex aspects of cognitive ToM abilities as highlighted by a correlation analysis, we failed to observe any comprehension difficulties in patients who performed poorly on simple cognitive ToM tasks, which suggests that AD truly affects cognitive ToM.
\end{abstract}

Keywords: Alzheimer's disease; Theory of mind; Emotions; Social cognition 


\section{Introduction}

"Theory of mind" (ToM) is a complex, high-level mental function that allows the independent mental states of self and others to be inferred. More specifically, this "mindreading" ability enables us to deduce the cognitive and emotional states (thoughts, feelings, beliefs, intentions, or desires) of other people from their attitudes and thus to anticipate and interpret their behaviors. ToM therefore appears to be intrinsically related to social cognition, crucially enabling us to understand and predict behaviors in everyday life. There is a growing body of evidence from both neuroimaging (Brunet et al., 2000; Fletcher et al., 1995; Gallagher et al., 2000; Völlm et al., 2006) and brain lesion studies (Channon \& Crawford, 2000; Gregory et al., 2002; Rowe et al., 2001; Shamay-Tsoory et al., 2005; Snowden et al., 2003; Stone et al., 1998; Stuss et al., 2001) that the frontal lobes, particularly the medial part of this region, play a pivotal role in ToM (Lee et al., 2010; Roca et al., 2011). Not surprisingly, therefore, ToM has been found to be impaired in the frontal variant of frontotemporal dementia (fv-FTD) even in the early stages of disease (Adenzato et al., 2010; Gregory et al., 2002; Lough et al., 2001; Lough et al., 2006; Snowden et al., 2003). This impairment may explain the changes in personality and social behavior that characterize this pathology.

Furthermore, several authors have shown that other cognitive functions, involved in goal-directed behavior, such as shifting, inhibition, updating or planning processes, contribute to ToM processing and may thus be responsible for its impairment. This has been invoked to explain ToM deficits not just in FTD but also in healthy aging (Duval et al., 2011; German \& Hehman, 2006). Surprisingly, only a handful of studies have looked at ToM abilities in other neurodegenerative diseases such as Alzheimer's disease (AD). However, in AD, frontal cortex abnormalities have been shown (see, for example, Fouquet et al., 2009) and executive dysfunction has also been demonstrated (Amieva et al., 2004). Thus, we can legitimately ask if these impairments have an impact on ToM. Furthermore, even at an early stage, AD patients sometimes exhibit behavioral disturbances in addition to cognitive impairment. Depression, apathy and anxiety are the most frequent and severe behavioral disturbances, whereas the more advanced stages are marked by a significant increase in delusions and irritability (Serra et al., 2010). Some of these disturbances may be explained, at least in part, by ToM deficits, as has been suggested in fv-FTD (Kipps et al., 2009). To our knowledge, García-Cuerva et al. (2001) were the first to assess ToM in AD, using a second-order false belief task in which individuals were asked to infer one character's belief about another character's belief. These 
authors showed that only $12 \mathrm{AD}$ patients out of 34 successfully completed the task and that these patients displayed less severe deficits in tests of verbal memory, verbal comprehension, abstract thinking, and naming than those who failed the task. Gregory et al. (2002) compared AD patients with fv-FTD patients using a first-order false belief task, a second-order false belief task and a faux pas test (detection of a social blunder or lack of tact in a scenario). In contrast to the patients with fv-FTD who performed poorly on all the ToM tests, the only task in which the AD group's performance was impaired was the second-order false belief task, which placed heavy demands on working memory. For their part, Zaitchik et al. (2004) used a first-order false belief task comprising four stories illustrated by simple line drawings. AD patients performed significantly more poorly than controls, but their performance was also found to be impaired in a control task which used similar stories but did not involve beliefs. Thus, all three studies would suggest that there is no genuine ToM deficit in AD patients, but that impaired performance on ToM tasks is secondary to other cognitive deficits. This idea is supported by several studies, in addition to that of Gregory et al. (2002), which have demonstrated that the ability to attribute beliefs remains intact in first-order, but not secondorder tasks (Castelli et al., 2011; Fernandez-Duque et al., 2009; Zaitchik et al., 2006). Similarly, using a cartoon task that required participants to process the psychological cause of events, Verdon et al. (2007) highlighted a deficit in attribution of intention in AD patients.

All the above studies focused on cognitive states, that is, on the beliefs, thoughts or intentions that correspond to what authors have called "cognitive ToM" (Coricelli, 2005), as opposed to "affective ToM", which allows us to represent the affective states, emotions or feelings of others in our minds. The latter can be tested using an expressive face recognition test or the Reading the Mind in the Eyes test (Baron-Cohen et al., 2001). To our knowledge, only Gregory et al. (2002), Zaitchik et al. (2006), Henry et al. (2009) and Castelli et al. (2011) have administered an affective ToM task to AD patients. While Gregory et al. (2002) found that early-stage $\mathrm{AD}$ patients had no difficulty inferring emotions from faces using the Eyes test, Henry et al. and Castelli et al. concluded that affective ToM is impaired in AD. This discrepancy could be due to the fact that in the former study, patients had to choose the right answer from only two words, whereas in the other two, there were four words to choose between. Finally, using four stories illustrated by simple line drawings, Zaitchik et al. (2006) showed that the ability to infer emotions is preserved in both first-order and second-order conditions, in contrast to the ability to infer beliefs (see above). According to the authors, this may be due to a lower demand in the emotion condition. 
Overall, the data available on ToM in $\mathrm{AD}$, albeit scant, appear to be relatively consistent, highlighting impaired performance only in the complex tasks, which suggests that deficits emerge when the task requires the intervention of cognitive functions that are disturbed in $\mathrm{AD}$, such as reasoning, memory or understanding, and do not stem from a primary deficit of ToM. However, as we have indicated, such studies are scarce, especially those exploring both cognitive and affective ToM (Castelli et al., 2011; Gregory et al., 2002; Zaitchik et al., 2006) and it remains unclear whether deficits are only due to other cognitive functions or if ToM itself is really disturbed, at least in the moderate stage of the disease. It may be that the most frequently employed version of the task (second-order false belief) used to assess ToM in the previous studies is too complex to avoid the intervention of executive functions, reasoning or memory. It is also worth noting that this task features only a limited number of stories (1-4). Moreover, although the involvement of other cognitive functions has been systematically invoked, the association between ToM and other cognitive functions has yet to be fully explored.

The aim of this study was to investigate changes in both the cognitive and affective dimensions of ToM in AD using a battery of tasks designed to overcome memory and comprehension difficulties of the patients. Our second objective was to gauge the extent to which the effects of $\mathrm{AD}$ on different aspects of ToM are linked to the impairment of working memory and executive functions.

\section{Methods}

\subsection{Participants}

Sixteen AD patients (5 men and 11 women, age range 73-83 years, mean 78.1 \pm 2.6 years) and 15 healthy controls (HC; 3 men and 12 women, age range 72-81 years, mean 76.4 \pm 3.2 years) were recruited for this study. All participants were French native speakers and had a minimum level of education equivalent to the now obsolete 'certificat d'études primaires', a school-leaving certificate that pupils generally took at $\sim 14$ years, following seven years of primary education. None of the participants had a history of alcoholism, head trauma, or neurological or psychiatric illness. The Mattis Dementia Rating Scale (MDRS; Mattis, 1976) was used to assess the general cognitive functioning of each participant.

All the AD patients met the National Institute of Neurological and Communicative Disorders and Stroke and the Alzheimer's Disease and Related Disorders Association (NINCDS-ADRDA) criteria for probable Alzheimer's disease (McKhann et al., 1984). They 
underwent a neurological examination including standard MRI scans and an extensive routine neuropsychological assessment. For each patient, the selection was made according to a codified procedure, in French approved centers whose main activity is the diagnosis and follow-up of patients suffering from neurodegenerative disorders. Neuropsychologists and speech therapists contributed to the diagnosis. Patients had to be capable of fully understanding instructions and/or performing the neuropsychological assessment. The patients' mean score on the Mini-Mental State Examination (MMSE; Folstein et al., 1975) was $21.5( \pm 3.4)$.

\subsection{Theory of mind tests}

In order to perform consistent measures of ToM within the AD patient group, we administered a series of assessment tasks that were designed to probe both the cognitive and the affective dimensions of ToM. More specifically, we used classic ToM tasks, adapting them in order to reduce cognitive load, notably memory, language and executive function demands. Some of these tasks were recently used in semantic dementia (Duval, Bejanin et al., 2012). Cognitive ToM was assessed by means of preference judgment (PJ) and false belief (FB) tasks. Affective ToM was assessed via a modified version of the Reading the Mind in the Eyes test (Baron-Cohen et al., 1997). We checked that participants fully understood each ToM task and, where necessary, provided aids to comprehension, mainly in the form of a glossary or the rewording of sentences. FB test contained a control/comprehension condition to determine whether any poor performance on the participants' part was truly due to a deficit in mental state inference.

\subsubsection{Preference judgment task}

This task, inspired by Snowden et al. (2003), assessed the ability to judge another person's preference based on gaze direction, minimizing executive demands. The material consisted of 20 cards, each showing the cartoon outline of a face, positioned centrally, and four colored pictures of items belonging to a single category, one in each of the four corners of the card. The gaze was directed towards one of the four pictures. Five object categories were used: cartoon characters, fruit, houses, cars and watches. Participants were instructed to point to the picture on the card that the central face liked best. They were not given any feedback about their choices. If the participant selected the picture that matched the direction of the central face's gaze, one point was awarded. Scores were expressed as a percentage of correct responses. To dismiss the possibility that visuospatial difficulties were the cause of the errors, cards for which the participant made incorrect choices were presented once again and 
he has to point to the picture that the central face was looking at. In the second part of the test, the cards were presented again, this time devoid of the faces, and participants had to indicate which of the four pictures on each one was their personal favorite. Errors in the first part of the test were coded as "favorite" if participants proved to have chosen their personal favorites, "perseveration" if they pointed to an item in the same position as the one they had selected immediately beforehand, and "random" if an incorrect choice did not fit into either of the above categories.

\subsubsection{False belief task}

We used an FB task consisting of an original visual-and-verbal test based on FB cartoon tasks such as "Sally and Ann" (Wimmer \& Perner, 1983). It was made up of 13 short comic strips that had been dreamed up within our laboratory, illustrating stories of everyday situations. Each comic strip comprised three pictures, each accompanied by a short written description which gave rise to one of the characters entertaining a false belief about the true state of affairs (for an example, see Fig. 1) To reduce cognitive load, the pictures and written descriptions remained visible throughout. The stories were all based on the same principle: 1) they described a situation involving a character who becomes aware of a certain piece of information, 2) unbeknownst to the character, the situation then changes. After studying each comic strip, participants were asked about the character's belief (i.e., a belief based on only partial knowledge of the facts). In order to answer the belief question correctly in a twoalternative forced choice, participants had to be able to understand that another person can hold a belief which may be mistaken. Five of the cartoons that involved first-order representations ("x thinks that...") were presented in a first time and in a second time participants had to answer to eight second-order ones ("x thinks that y thinks that..."). We inserted an interference task (span task; see below) between the first- and second-order FB conditions and between the ToM tasks.

Each correct answer was scored one point. Participants were not given any feedback about their choices. Performances were expressed as a percentage of correct responses for each condition.

To assess the participants' story comprehension, once they had completed the two FB conditions, they were presented with the same stories again, this time with a reality question. In this comprehension task, the two alternatives were the same as in the FB conditions. Each correct answer was scored one point and scores were expressed as a percentage of correct responses. 
Figure 1. Example of the stimuli and the questions used in the first- and second-order false belief task

\subsubsection{Reading the Mind in the Eyes test}

The Eyes test was derived from the material and procedure used by Baron-Cohen et al. (1997) to assess the attribution of affective mental states (or affective ToM) by requiring participants to make inferences about the affective and motivational states of others on the basis of a picture of their eyes. The test consisted of 36 black-and-white photographs showing the eye regions of 36 different faces, either male or female. Each picture had two mental state terms printed below it (e.g. terrified/regretful). Participants were asked to decide which of two words best reflected what the person in the picture was thinking or feeling. To minimize comprehension difficulties, a glossary with synonyms and a concrete example of the use of each word were available to participants. In addition, an example was used at the beginning of the task to familiarize participants with the material. Each correct response was scored one point and scores were expressed as a percentage of correct responses.

\subsection{Supplementary neuropsychological tests}

The AD patients also underwent standardized neuropsychological tests to assess working memory (backward digit span) and executive functions. Inhibition was measured according to the number of errors in the color-naming interference condition of the Stroop Test (Godefroy \& GREFEX, 2008) and strategic processes by means of the phonemic fluency tasks (Cardebat et al., 1990). The time it took participants to complete the color naming condition of the Stroop Test (Godefroy \& GREFEX, 2008) was assumed to reflect information processing speed.

\subsection{Statistical methods}

All statistical analyses were performed using StatSoft Statistica 9.0. The threshold of significance was set at $p=.05$ (one-tailed). Unpaired two-sample $t$-tests were carried out to compare the demographic and general neuropsychological data of the two groups. A Group $\left(\mathrm{HC}\right.$ vs. AD) $x$ Order $\left(1^{\text {st }}\right.$ and $\left.2^{\text {nd }}\right)$ factorial ANOVA on the FB scores. Follow-up post hoc comparisons were conducted with Tukey's Honestly Significant Difference (HSD) test. The performances of both groups in the comprehension condition of the FB task, and on the PJ and Eyes tests were compared by means of unpaired two-sample $t$-tests. In addition, Pearson's chi-square goodness-of-fit test was used to test whether the AD patients had made specific errors on the PJ task. 
In the $\mathrm{AD}$ group, correlation analyses (Pearson's correlation coefficient) were conducted between the different scores of the ToM tasks (PJ, first- and second-order conditions of the FB and Eyes test scores), between the Comprehension and both the first- and second-order scores of the FB task, and finally between the ToM task scores and the level of education and neuropsychological data.

\section{Results}

\subsection{Demographic and general clinical data (Table 1)}

The two groups were well matched in terms of age and educational level. It is interesting to note that both $\mathrm{HC}$ and $\mathrm{AD}$ patients presented a low level of education, which is standard for France's older rural population. As expected, performances on the MDRS, Stroop interference, phonemic fluency tests were significantly poorer in the AD group than in the HC group.

Table 1 - Demographic data and performances on general neuropsychological tests

\begin{tabular}{llll}
\hline & HC participants (15) & AD patients (16) & Unpaired $t$-test $p$ value \\
\hline Age (years) & $76.4( \pm 3.2)$ & $78.1( \pm 2.6)$ & .11 \\
Education (years) & $7.5( \pm 1.6)$ & $7.7( \pm 2.5)$ & .77 \\
MDRS & $141( \pm 4.4)$ & $121.1( \pm 7.2)$ & $<.001$ \\
Backward Digit Span & - & $3.5( \pm 1.0)$ & - \\
Stroop Interference & $2.1( \pm 2.3)$ & $20.2( \pm 14.9)$ & $<.001$ \\
Phonemic Fluency & $18.5( \pm 6.8)$ & $13.6( \pm 6.0)$ & $<.05$ \\
\hline
\end{tabular}

$\mathrm{AD}=$ Alzheimer's disease, MDRS = Mattis Dementia Rating Scale, $\mathrm{HC}=$ Healthy controls.

\subsection{Preference judgment task}

For the PJ task, a $t$-test indicated a significant difference between the AD and HC groups $\left[t(29)=2.60, p<.01, \eta^{2}=0.19\right]$, with lower performances for the AD patients $(15.2 \pm 6.4)$ relative to controls (19.6 \pm 0.9 ). For $\mathrm{AD}$ and $\mathrm{HC}$, none of the errors in judging preference was related to an error in determining the direction of the gaze. For the AD group, the three types 
of errors (favorite, perseveration and random) represented 37\%, 24\% and 39\% of errors, respectively. This distribution did not vary $\left[X^{2}(2)=2.39, p=.30\right]$.

\subsection{False-belief task}

For the comprehension condition of the FB task, a $t$-test failed to indicate a significant difference between the two groups $\left[t(29)=-0.93, p^{<.18}, \eta^{2}=0.03\right]$. The AD patients scored well in the comprehension condition (mean $86.1 \% \pm 8.5$ ).

For the ToM condition, the ANOVA showed a significant Group effect $[F(1,58)=14.26$, $\left.p<.001, \eta^{2}=0.29\right]$ and a significant Order effect $\left[F(1,58)=14.89, p<.001, \eta^{2}=0.39\right]$ but the Group x Order interaction effect was not significant $\left[F(1,58)=0.34, p=.56, \eta^{2}=0.01\right]$. Post hoc analyses indicated that there was a significant difference between the AD and HC groups $(p<.02)$, with lower performances for the AD group in the first-order condition, but a nonsignificant difference in the second-order condition $(p=.12)$. There was a significant difference between the first- and second-order conditions in the HC group ( $p<.02)$, with lower performances in the second-order condition, but the difference was not significant in the AD group ( $p=.10)$ (Fig. 2). It is worth noting that the AD group's mean performance in the second-order condition was near chance level (mean 52.3\% \pm 18.4 ).

Figure 2. Performances (mean and standard deviation) on the first- and second-order false belief task for control (HC) and Alzheimer's disease (AD) groups.

\subsection{Reading the Mind in the Eyes test}

For the Eyes test, the AD patients' performances (mean 67.9 \pm 12.0 ) were lower than those of the controls (mean $75.0 \pm 8.4$ ). A $t$-test indicate a significant difference between the $\mathrm{AD}$ and $\mathrm{HC}$ groups $\left[t(29)=1.9, p<.04, \eta^{2}=0.11\right]$.

\subsection{Correlation analyses}

Regarding the correlation analyses between the ToM scores in the AD group, the only significant correlation was between the first-order FB condition and PJ scores $(r=0.51$; $p<.03)$. The correlations between Comprehension and both the first- and second-order conditions of the FB were nonsignificant ( $r=0.24 ; p=.18$ and $r=-0.15 ; p=.29$, respectively).

The correlation analyses between level of education, the neuropsychological data and performances on the ToM tasks are reported in Table 2 . We found significant correlations between education and all the ToM scores (all $p<.05$ ), between the first-order FB score and the MDRS $(p<.01)$, between the second-order FB score and the backward digit $\operatorname{span}(p<.02)$, 
Stroop interference $(p<.02)$ and phonemic fluency scores $(p<.01)$, and between the Eyes test and MDRS scores $(p<.02)$.

Table 2 - Pearson correlation coefficients between level of education, neuropsychological data and performances on the ToM tasks for the AD group

\begin{tabular}{lllll}
\hline ToM tasks & PJ & $1^{\text {st }}$-order FB & $2^{\text {nd }}$-order FB & Eyes test \\
\hline Education (years) & $0.47^{*}$ & $0.53^{*}$ & $0.57^{*}$ & $0.43^{*}$ \\
MDRS & 0.35 & $0.58^{*}$ & 0.19 & $0.56^{*}$ \\
Backward Digit Span & 0.27 & 0.33 & $0.54^{*}$ & -0.15 \\
Stroop Interference & -0.22 & -0.09 & $-0.52^{*}$ & -0.18 \\
Phonemic Fluency & 0.27 & 0.38 & $0.57^{*}$ & 0.42 \\
\hline
\end{tabular}

$\mathrm{FB}=$ False belief, $\mathrm{PJ}=$ Preference judgment. $*$ indicates a significant correlation coefficient, $p<.05$.

\section{Discussion}

We observed cognitive and affective ToM deficits in the mild to moderate AD patients even for basic aspects of social cognition such as gaze information processing. The few studies to have explored ToM in $\mathrm{AD}$ up to now reported deficits that were mostly confined to the more complex tasks involving inference of cognitive mental states (second-order FB) while emotional inference was found to be relatively preserved. As the classic second-order FB task places heavy demands on language, working memory and episodic memory, some authors have suggested that the ToM deficits reported in $\mathrm{AD}$ are a consequence of general cognitive impairment and comprehension difficulties. Using tasks designed to circumvent these patients' difficulties, we reported impaired performances in both cognitive and affective ToM in mild to moderate AD patients with preserved comprehension of the FB stories.

The ability to use information about a person's mental states conveyed by gaze is one of the basic aspects of social cue decoding. Impaired preference judgment based on gaze has previously been reported in patients with frontotemporal lobar degeneration, who were found to base their responses on their own personal favorites (Duval, Bejanin et al., 2012; Snowden et al., 2003). To the best of our knowledge, the recent study by Castelli et al. (2011) is the 
only one to have used this task in AD. These authors also reported significant differences between the control and AD groups and, in their Discussion, noted that patients said their choices were driven by their own personal preferences, although these assertions were not put to test. In the present study, therefore, specific questions were asked in order to highlight the nature of the incorrect responses. We observed that incorrect selections corresponding to the patients' own personal preferences were no more frequent than perseverative or random errors. In addition, "favorite" errors were not the predominant error type for any of the patients. Thus, while both frontotemporal and AD patients present deficits in their preference judgment performances, differences in their incorrect selections suggest differences in the nature of these deficits.

The preference judgment test minimizes the demands placed on memory and executive functions and does not require any active mental manipulation or integration of information. We can therefore assume that the impaired performances of AD patients on this task occurred independently of any memory and executive disorders. Moreover, we did not observe any significant correlations between performances on this test and working memory or executive measures. Although visuospatial deficits might have been involved in this impairment, this seems unlikely, given that none of them failed to decide which of the four pictures the central character was looking at. This attests to the preservation of gaze perception in mild to moderate $\mathrm{AD}$, but difficulty in using this information to assess another person's preferences.

We also observed impaired performances in more complex cognitive ToM dimensions. FB assessments frequently use verbal stories and questions. This presentation modality may contribute to $\mathrm{AD}$ patients' poor performances owing to their working memory and language deficits. Taking these deficits into account, we chose to use simple visual and verbal stories instead, and we made a point of ensuring that the patients understood them. To that end, the examiner reformulated the key information in the stories as much as necessary.

Using this methodology, our findings concerning first-order FB take previous results one step further. To our knowledge, this is the first time that first-order FB deficits have been brought to light in mild to moderate AD using a task designed to sidestep patients' comprehension difficulties. It is important to note that we did not find any significant correlation between the FB comprehension score and either the first- or the second-order FB inferences in the $\mathrm{AD}$ group. In addition, we showed that difficulties in inferring first-order beliefs increase as the disease progresses, as indicated by the correlation with the MDRS score. Interestingly, those previous studies that failed to report differences between AD 
patients and controls in first-order FB tasks were conducted in patients in an earlier stage of the disease, as attested by their mean MMSE scores (Castelli et al., 2011; Fernandez-Duque et al., 2009; Gregory et al., 2002; Zaitchik et al., 2006). In addition, methodological characteristics may be responsible for this apparent discrepancy, in that some of the tasks used may have been inadequate, as suggested by the ceiling effects observed in some of these studies. Finally, differences between these studies and ours regarding the participants' level of education need to be considered all the more so since we observed significant correlations between both first- and second-order FB inferences and education (number of years).

Regarding second-order FB, our results confirm previous reports in AD (Castelli et al., 2011; Fernandez-Duque et al., 2009; Garcia-Cuerva et al., 2001; Gregory et al., 2002; Zaitchik et al., 2006). Results of the Group by Order ANOVA (i.e. significant Group and Order effects and a non significant interaction) indicated deficits both in the first- and the second-order FB conditions in AD. We did not report larger deficits in the second-order FB condition than in the first-order one. Post-hoc analyses showed significant differences between $\mathrm{AD}$ and $\mathrm{HC}$ groups in the first-order, but not in the second-order condition. This is problaby due to floor effects in the second-order condition where AD patients performed at the level of chance. In addition, a direct aging effect on second-order ToM abilities in healthy subjects (Duval et al., 2011) may have contributed to reduce the difference between AD and HC performances.

Some authors have hypothesized that deficits in second-order FB are due to the task's cognitive processing demands, rather than to reasoning about mental states. We did indeed find significant correlations between second-order FB inference and both inhibition (Stroop test) and the ability to manipulate verbal information, as measured by phonemic verbal fluency and the backward digit span. This finding reasserts, if any confirmation were needed, the link between executive functions and ToM (see Aboulafia-Brakha et al., 2011, for review). In addition, it underlines the specific role of working memory in second-order FB tasks, which require the representation and manipulation of doubly embedded clauses.

Turning to the finding in affective ToM, we reported a significant effect of AD and we observed greater difficulties related to disease severity, as indicated by the significant correlation with the MDRS score. Of the very few studies to have used the Eyes test in AD, Gregory et al. (2002) reported intact performances in very mild AD patients, while Castelli et al. (2011) observed deficits in mild to moderate patients. Moreover, in a longitudinal study of 19 AD patients, Lavenu and Pasquier (2005) reported a decrease in the recognition of facially 
expressed emotion as dementia progressed. In the Eyes test, some of the words are complex and participants have to choose between close concepts. In $\mathrm{AD}$, semantic deterioration starts with the disappearance of the fine-grained differences between close semantic representations (Laisney et al., 2011). As with neutral concepts, emotional ones seem to lose some of their distinctive attributes in AD (Giffard et al., 2009). However, we can assume that the glossary we made available in the Eyes test allowed the AD patients to overcome their comprehension difficulties.

Affective ToM seemed affected to a lesser degree than the cognitive dimension. It is important to note that, unlike the Eyes test, which simply requires mental states to be decoded on the basis of immediately available information, FB task need mental state reasoning skills to be implemented in order to produce reliable inferences about other people's beliefs. The interesting point is that we also observed an $\mathrm{AD}$ effect in the PJ test, which consists in identifying the focus of other people's attention based on the direction of their gaze, and again only requires the decoding of mental states.

Interestingly, we reported significant correlations between the level of education and all of the AD patients' ToM performances. It is well known that education can influence cognitive performance in both verbal and non verbal measures. To our knowledge in the literature on ToM in adults, education is taken into account by matching groups of subjects but any study was dedicated to the effect of this variable on the performances. Maylor et al. (2002) reported a significant correlation between education and ToM performances in ageing. The significant correlations that we reported in AD suggest a general effect of crystallized intelligence on ToM but could also be related to the ability for compensation allowed by cognitive reserve.

ToM abilities are related to a brain network composed of the medial prefrontal cortex, posterior cingulate/precuneus, and bilateral temporal parietal junction (Kemp et al., 2012, for review). Temporal parietal junction and posterior cingulate alterations are commonly reported in the AD literature (Villain et al., 2010) and more recent findings indicated a ventro-medial prefrontal region hypometabolism in the first stages of AD (Fouquet et al., 2009). This could contribute to the social dysfunction observed in mild to moderate AD. However, to date any study was dedicated to the specific relationships between ToM difficulties and brain alterations in $\mathrm{AD}$.

Further research is needed to pinpoint the exact stage in the disease at which the ToM disorder appears, as it is crucial to be able to identify such deficits in AD. Misunderstanding 
of social situations and misinterpretation of the interests and beliefs of others may drive patients to distance themselves or produce anxiety, irritability and even aggressive behaviors. We need to clarify the relationship between ToM disorders and behavioral changes in AD in order to give caregivers advice on how to manage interactions with their charges.

\section{References}

Aboulafia-Brakha T., Christe B., Martory M.D., \& Annoni J.M. (2011). Theory of mind tasks and executive functions: a systematic review of group studies in neurology. Journal of.Neuropsychology, 5, 39-55.

Adenzato, M., Cavallo, M., \& Enrici, I. (2010). Theory of mind ability in the behavioural variant of frontotemporal dementia: An analysis of the neural, cognitive, and social levels. Neuropsychologia, 48, 2-12.

Amieva, H., Phillips, L. H., Della Sala, S., \& Henry, J. D. (2004). Inhibitory functioning in Alzheimer's disease. Brain, 127, 949-964.

Baron-Cohen, S., Jolliffe, T., Mortimore, C., \& Robertson, M. (1997). Another advanced test of theory of mind: Evidence from very high functioning adults with autism or Asperger syndrome. Journal of Child Psychology and Psychiatry and Allied Disciplines, 38, 813822.

Baron-Cohen, S., Wheelwright, S., Hill, J., Raste, Y., \& Plumb, I. (2001). The "Reading the Mind in the Eyes" Test revised version: A study with normal adults, and adults with Asperger syndrome or high-functioning autism. Journal of Child Psychology and Psychiatry and Allied Disciplines, 42, 241-251.

Brunet, E., Sarfati, Y., Hardy-Baylé, M. C., \& Decety, J. (2000). A PET investigation of the attribution of intentions with a nonverbal task. NeuroImage, 11, 157-166.

Cardebat, D., Doyon, B., Puel, M., Goulet, P., \& Joanette, Y. (1990). Formal and semantic lexical evocation in normal subjects. Performance and dynamics of production as a function of sex, age and educational level. Acta Neurologica Belgica, 90, 207-217.

Castelli, I., Pini, A., Alberoni, M., Liverta-Sempio, O., Baglio, F., Massaro, D. Marchetti, A., \& Nemni, R. (2011). Mapping levels of theory of mind in Alzheimer's disease: A preliminary study. Aging \& Mental Health, 15, 157-168. 
Channon, S., \& Crawford, S. (2000). The effects of anterior lesions on performance on a story comprehension test: Left anterior impairment on a theory of mind-type task. Neuropsychologia, 38, 1006-1017.

Coricelli, G. (2005). Two-levels of mental states attribution: From automaticity to voluntariness. Neuropsychologia, 43, 294-300.

Duval, C., Bejanin, A., Piolino, P., Laisney, M., Eustache, F., \& Desgranges, B. (2012). Theory of mind impairments in patients with semantic dementia. Brain, 135, 228-241.

Duval, C., Piolino, P., Bejanin, A., Eustache, F., \& Desgranges, B. (2011). Age effects on different components of theory of mind. Consciousness and Cognition, 20, 627-642.

Fernandez-Duque, D., Baird, J. A., \& Black, S. E. (2009). False-belief understanding in frontotemporal dementia and Alzheimer's disease. Journal of Clinical and Experimental Neuropsychology, 31, 489-497.

Fletcher, P. C., Happe, F., Frith, U., Baker, S. C., Dolan, R. J., Frackowiak, R. S. \& Frith, C.D. (1995). Other minds in the brain: A functional imaging study of "theory of mind" in story comprehension. Cognition, 57, 109-128.

Folstein, M. F., Folstein, S. E., \& McHugh, P. R. (1975). "Mini-mental state" - A practical method for grading the cognitive state of patients for the clinician. Journal of Psychiatric Research, 12, 189-198.

Fouquet, M., Desgranges, B., Landeau, B., Duchesnay, E., Mézenge, F., de La Sayette, V. Viader, F., Baron, J.C., Eustache, F., \& Chételat, G. (2009). Longitudinal brain metabolic changes from amnestic mild cognitive impairment to Alzheimer's disease. Brain, 132, 2058-2067.

Gallagher, H. L., Happe, F., Brunswick, N., Fletcher, P. C., Frith, U., \& Frith, C. D. (2000). Reading the mind in cartoons and stories: An fMRI study of "theory of mind" in verbal and nonverbal tasks. Neuropsychologia, 38, 11-21.

Garcia-Cuerva, A., Sabe, L., Kuzis, G., Tiberti, C., Dorrego, F., \& Starkstein, S. E. (2001). Theory of mind and pragmatic abilities in dementia. Neuropsychiatry, Neuropsychology, and Behavioral Neurology, 14, 153-158. 
German, T. P., \& Hehman, J. A. (2006). Representational and executive selection resources in "theory of mind": Evidence from compromised belief-desire reasoning in old age. Cognition, 101, 129-152.

Giffard, B., Laisney, M., Eustache, F., \& Desgranges, B. (2009). Can the emotional connotation of concepts modulate the lexico-semantic deficits in Alzheimer's disease? Neuropsychologia, 47, 258-267.

Godefroy, O., \& GREFEX (2008). Fonctions exécutives et pathologies neurologiques et psychiatriques - Evaluation en pratique clinique. Marseilles: Solal.

Gregory, C., Lough, S., Stone, V., Erzinclioglu, S., Martin, L., Baron-Cohen, S. \& Hodges, J.R. (2002). Theory of mind in patients with frontal variant frontotemporal dementia and Alzheimer's disease: Theoretical and practical implications. Brain, 125, 752-764.

Henry, J. D., Rendell, P. G., Scicluna, A., Jackson, M., \& Phillips, L. H. (2009). Emotion experience, expression, and regulation in Alzheimer's disease. Psychology and Aging, 24, 252-257.

Kemp J., Despres O., Sellal F., \& Dufour A. (2012). Theory of Mind in normal ageing and neurodegenerative pathologies. Ageing Research Review, 11, 199-219.

Kipps, C. M., Mioshi, E., \& Hodges, J. R. (2009). Emotion, social functioning and activities of daily living in frontotemporal dementia. Neurocase, 15, 182-189.

Laisney, M., Giffard, B., Belliard, S., de La Sayette, V., Desgranges, B., \& Eustache, F. (2011). When the zebra loses its stripes: Semantic priming in early Alzheimer's disease and semantic dementia. Cortex, 47, 35-46.

Lavenu, I., \& Pasquier, F. (2005). Perception of emotion on faces in frontotemporal dementia and Alzheimer's disease: A longitudinal study. Dementia and Geriatric Cognitive Disorders, 19, 37-41.

Lee T.M., Ip A.K., Wang K., Xi C.H., Hu P.P., Mak H.K., Han S.H. \& Chan C.C. (2010). Faux pas deficits in people with medial frontal lesions as related to impaired understanding of a speaker's mental state. Neuropsychologia, 48, 1670-1676. 
Lough, S., Gregory, C., \& Hodges, J. R. (2001). Dissociation of social cognition and executive function in frontal variant frontotemporal dementia. Neurocase, 7, 123-130.

Lough, S., Kipps, C. M., Treise, C., Watson, P., Blair, J. R., \& Hodges, J. R. (2006). Social reasoning, emotion and empathy in frontotemporal dementia. Neuropsychologia, 44, 950-958.

Mattis, S. (1976). Mental status examination for organic mental syndrome in the elderly patient. In L. Bellack \& T. Katasu (Eds.), Geriatric psychiatry: A handbook for psychiatrists and primary care physicians (pp. 77-120). New York: Grune and Stratton.

Maylor E.A., Moulson J.M., Muncer A.M., \& Taylor L.A. (2002). Does performance on theory of mind tasks decline in old age? British Journal of Psychology, 93, 465-485.

McKhann, G., Drachman, D., Folstein, M., Katzman, R., Price, D., \& Stadlan, E. M. (1984). Clinical diagnosis of Alzheimer's disease: Report of the NINCDS-ADRDA Work Group under the auspices of Department of Health and Human Services Task Force on Alzheimer's Disease. Neurology, 34, 939-944.

Roca M., Torralva T., Gleichgerrcht E., Woolgar A., Thompson R., Duncan J., \& Manes F. (2011). The role of Area 10 (BA10) in human multitasking and in social cognition: a lesion study. Neuropsychologia, 49, 3525-3531.

Rowe, A. D., Bullock, P. R., Polkey, C. E., \& Morris, R. G. (2001). "Theory of mind" impairments and their relationship to executive functioning following frontal lobe excisions. Brain, 124, 600-616.

Serra, L., Perri, R., Fadda, L., Padovani, A., Lorusso, S., Pettenati, C. Caltagirone, C., \& Carlesimo, G.A. (2010). Relationship between cognitive impairment and behavioural disturbances in Alzheimer's disease patients. Behavioural Neurology, 23, 123-130.

Shamay-Tsoory, S. G., \& Aharon-Peretz, J. (2007). Dissociable prefrontal networks for cognitive and affective theory of mind: A lesion study. Neuropsychologia, 45, 30543067.

Shamay-Tsoory, S. G., Tomer, R., Berger, B. D., Goldsher, D., \& Aharon-Peretz, J. (2005). Impaired "affective theory of mind" is associated with right ventromedial prefrontal 
damage. Cognitive and Behavioral Neurology: Official Journal of the Society for Behavioral and Cognitive Neurology, 18, 55-67.

Snowden, J. S., Gibbons, Z. C., Blackshaw, A., Doubleday, E., Thompson, J., Craufurd, D. Foster, J., Happé, F., \& Neary, D. (2003). Social cognition in frontotemporal dementia and Huntington's disease. Neuropsychologia, 41, 688-701.

Stone, V. E., Baron-Cohen, S., \& Knight, R. T. (1998). Frontal lobe contributions to theory of mind. Journal of Cognitive Neuroscience, 10, 640-656.

Stuss, D. T., Gallup, G. G., Jr., \& Alexander, M. P. (2001). The frontal lobes are necessary for "theory of mind". Brain, 124, 279-286.

Verdon, C. M., Fossati, P., Verny, M., Dieudonné, B., Teillet, L., \& Nadel, J. (2007). Social cognition: An early impairment in dementia of the Alzheimer type. Alzheimer Disease and Associated Disorders, 21, 25-30.

Villain N., Fouquet M., Baron J.C., Mezenge F., Landeau B., de La Sayette, V., Viader F., Eustache F., Desgranges B., \& Chételat G. (2010).Sequential relationships between grey matter and white matter atrophy and brain metabolic abnormalities in early Alzheimer's disease. Brain, 133, 3301-3314.

Völlm, B. A., Taylor, A. N., Richardson, P., Corcoran, R., Stirling, J., McKie, S. Deakin, J.F., \& Elliott, R. (2006). Neuronal correlates of theory of mind and empathy: A functional magnetic resonance imaging study in a nonverbal task. NeuroImage, 29, 90-98.

Wimmer, H., \& Perner, J. (1983). Beliefs about beliefs: Representation and constraining function of wrong beliefs in young children's understanding of deception. Cognition, 13, 103-128.

Zaitchik, D., Koff, E., Brownell, H., Winner, E., \& Albert, M. (2004). Inference of mental states in patients with Alzheimer's disease. Cognitive Neuropsychiatry, 9, 301-313.

Zaitchik, D., Koff, E., Brownell, H., Winner, E., \& Albert, M. (2006). Inference of beliefs and emotions in patients with Alzheimer's disease. Neuropsychology, 20, 11-20. 